\title{
Study on Stress Corrosion Characteristics of Drill Rod Joint Under Mechanical Effects
}

\author{
Youzhen Zhang $^{1 *}$, Zicheng zhong ${ }^{1}$, Peiying Mou $^{1}$, Junjie Shao ${ }^{1}$ and Ning Zhang ${ }^{1}$ \\ ${ }^{1}$ Xi' an Research Institute, China Coal Technology \& Engineering Group Corp., Xi'an, 710077, China
}

\begin{abstract}
Thanks to the complex underground environment that coal mines enjoy, drill rods for mining are vulnerable to corrosion during operation. To investigate the impact of the corrosion defects on the residual intensity of the drill rod, a relational expression of stress versus corrosion rate in the conditions of uniform and local corrosions is deduced based on the theory on mechanochemical effects; building on this, a spherical corrosion defect is developed in the joint of a $\varphi 73 \mathrm{~mm}$ drill rod while it is exerted with a make-up torque, an axial force and a bending moment. So it is found that, when the corrosion defect is under pressure, the bending moment plays a certain role to inhibit its increase, however when it is under tension, the bending moment plays the role to drive its increase so as to quicken its corrosion rate while the impact of the change in the corrosion detect depth is much greater than that of the radius. The result from the research provides a basis to evaluate the residual intensity of the drill rod and theoretical basis to protect drill rods from corrosion.
\end{abstract}

\section{Introduction}

As one of important tools in underground drilling of coal mines, the drill rod is a main component that transmits and bears forces during drilling. Recent years have been seeing progress in the underground coal-mine drilling technology and increased capability of the drilling machines and tools, and the research on structure and intensity of drill rods in various galleries has made some advances.

Drill rods used in gallery drilling mainly include conventional outer-flat drill rod, central cable drill rod, and wireline coring drill rod. Owing to the environmental specificities in underground coal mines, high temperature and humidity, corrosive gases such as $\mathrm{CO}_{2}, \mathrm{H}_{2} \mathrm{~S}, \mathrm{SO}_{2}$, and corrosive water, among other environmental factors in coal mines, will corrode the drill rods, and drill rod joints are much vulnerable to damage under joint action of stress and the corrosive environment, adverse to safety during drilling operation. Subjected to axial force, bending moment and torsional stress, etc., drill rods are required to be strong enough, reliable in operation and long in service life. According to statistics in the technical area of petroleum drilling and production, about $70 \%$ of drill rod failures are caused by corrosion fatigue[1], leading to incalculable financial loss.

Because of the action of corrosive medium, the stress corrosion in drill rods[2-3] includes uniform corrosion and local corrosion. Local corrosion will leave stress concentrated at the corrosion defect of the drill rod, and thanks to the bending moment[4-5], the stress at the corrosive place ramps up, thus accelerating the corrosion. Hence, studying the impact of the bending moment and the corrosion-defect-related parameters on stress in the corrosion defect of drill rod joints is critical to protect drill rods from corrosion and improve the safety of drilling.

\section{Relation of Stress versus Corrosion Rate}

\subsection{Relation of Stress versus Corrosion Rate under Uniform Corrosion}

During practical use of drill rods, its stress condition changes in the process of use or in the condition of steady external load, because the section changes due to drill rod being damaged from corrosion, and concentration of stress generated would reinforce the mechanochemical corrosion leading the bearing ability of drill rods to decrease quicker.

It is typically understood that the drill rod, in practical use, is mainly experiencing torque, axial force and bending moment, and it is known from the formula on material mechanics:

The axial force:

$$
\sigma_{\mathrm{z}}=4 T /\left[\pi\left(D^{2}-\mathrm{d}^{2}\right)\right]+P(D+\mathrm{d}) /[4(D-\mathrm{d})]
$$

The torsional shear stress:

$$
\left.\tau_{\max }=16 M_{\mathrm{t}} \times 10^{-6} / \mid \pi D^{3}\left(1-(d / D)^{4}\right)\right\rfloor
$$

Where, $\mathrm{T}$ is the torque being exerted to the drill rod, $\mathrm{N} \cdot \mathrm{m}$; $\mathrm{P}$ is the axial force being exerted to it, $\mathrm{MPa}$; $\mathrm{Mt}$ is the bending moment being exerted to it, $\mathrm{N} \cdot \mathrm{m}$; D is the outer diameter of it, $\mathrm{mm}$; $\mathrm{d}$ is the inner diameter of it, $\mathrm{mm}$.

In the condition of uniform corrosion, in accordance with the theory on mechanochemical effect, it is assumed 
that the load just changes the drill rod's corrosion rate with no damage to corrosion features, and at this moment, under the action of the axial force and the bending moment, the increase of the depth of the drill rod's corrosion defect determines the change of stress there as shown in Formula (3).

$$
\frac{d \sigma}{d \mathrm{~s}}=\frac{d\left(\sigma_{z}+\tau_{\max }\right)}{d \mathrm{~s}}=\frac{4 T}{\pi\left[(D-\mathrm{s})^{2}-\mathrm{d}^{2}\right]}+\frac{P(D-\mathrm{s}+\mathrm{d})}{4(D-\mathrm{s}-\mathrm{d})}+\frac{16 M_{\mathrm{t}} \times 10^{-6}}{\pi(D-\mathrm{s})^{3}\left(1-(d /(D-\mathrm{s}))^{4}\right)}
$$

$$
\sigma_{1}-\sigma_{0}=\frac{4 T}{\pi\left[\left(D-\mathrm{s}_{1}\right)^{2}-\mathrm{d}^{2}\right]}+\frac{P\left(D-\mathrm{s}_{1}+\mathrm{d}\right)}{4\left(D-\mathrm{s}_{1}-\mathrm{d}\right)}+\frac{16 M_{\mathrm{t}} \times 10^{-6}}{\pi\left(D-\mathrm{s}_{1}\right)^{3}\left(1-\left(d /\left(D-\mathrm{s}_{1}\right)\right)^{4}\right)}-\frac{4 T}{\pi\left(D^{2}-\mathrm{d}^{2}\right)}-\frac{P(D+\mathrm{d})}{4(D-\mathrm{d})}-\frac{16 M_{\mathrm{t}} \times 10^{-6}}{\pi D^{3}\left(1-(d / D)^{4}\right)}
$$

In this paper, the change of thickness of the corrosion defect is used as the index of the corrosion rate, and the rate of thickness reduction equals to the corrosion rate which depends on the acceleration process of anode dissolution caused by mechanic stress as shown in Formula (5).

$$
\begin{aligned}
& \frac{d s}{d t}=-v_{0} \exp \frac{V \sigma}{R T} \\
& \mathrm{t}_{1}=\frac{\left(\sigma_{1}-\sigma_{0}\right) \exp -\frac{V \sigma}{R T}}{\left\{\frac{4 T}{\pi\left[\left(D-\mathrm{s}_{1}\right)^{2}-\mathrm{d}^{2}\right]}+\frac{P\left(D-\mathrm{s}_{1}+\mathrm{d}\right)}{4\left(D-\mathrm{s}_{1}-\mathrm{d}\right)}+\frac{16 M_{\mathrm{t}} \times 10^{-6}}{\pi\left(D-\mathrm{s}_{1}\right)^{3}\left(1-\left(d /\left(D-\mathrm{s}_{1}\right)\right)^{4}\right)}-\frac{4 T}{\pi\left(D^{2}-\mathrm{d}^{2}\right)}-\frac{P(D+\mathrm{d})}{4(D-\mathrm{d})}-\frac{16 M_{\mathrm{t}} \times 10^{-6}}{\pi D^{3}\left(1-(d / D)^{4}\right)}\right\}}
\end{aligned}
$$
$\mathrm{T}$ is the absolute temperature, $\mathrm{K}$.
Where, $\mathrm{s}$ is the external corrosion's depth, mm.

Apply the integral deformation to the above Formula (3), from $\sigma 0$ (the pressure applied to the drill rod before corrosion starts) to $\sigma 1$ (the pressure applied to it after corrosion) for $\sigma$, and from 0 to $\mathrm{s} 1$ (the corrosion depth) for $\mathrm{s}$, and simplify the integral to get the Formula (4).
When $\sigma$ lis the stress when the drill rod fails, Formula (6) can be used to calculate the time length of the drill rod from uniform corrosion under the action of torque and bending moment.

\subsection{Relation of Stress versus Corrosion Rate under Local Corrosion}

There is a variety of corrosion that drill rods will suffer due to complex environments, however, in terms of the form of damage, there are two kinds of corrosion: general corrosion and local corrosion. Thanks to long exposure to external medium, chemical or electrochemical action occurs in the inner and outer walls of the drill rod, resulting in corrosion, and local corrosion prevails. Furthermore, local corrosion includes pitting, stress corrosion and electrochemical corrosion, etc.

For drill rods for mining, during its operation, in the case of stress generated, the stress will concentrate locally in the drill rod, accelerating the rate of local corrosion. It is assumed that the rate of local corrosion in drill rods is $\mathrm{K}$ times that of uniform corrosion (K: stress concentration factor).

The formula of the stress concentration coefficient $\mathrm{Kt}$ :

$$
K_{\mathrm{t}}=\text { Stress }_{M A X} / \text { Stress }_{A V G}
$$

The time length of failure is:

$$
\mathrm{t}_{2}=K_{\mathrm{t}} \mathrm{t}_{1}
$$

\section{Forces Borne by Connecting Threads}

The tightening torque will tighten the connection between inner and outer threads and generate a pressing force at the shoulder. The pressing force acts against the outer thread
Where, $\mathrm{V}$ is the steel's gram molecular volume; v0 is the rate of the uniform corrosion in the tubular body when no stress is applied; $\mathrm{R}$ is the universal gas constant, $8.314 \mathrm{~mol}-1 \cdot \mathrm{K}-1 ; \sigma$ is the absolute value of stress in metal in the case of unidirectional loading, under the elastic limit;

Reorganize formulas (4) and (5), and simplify the

to generate the tensile load and against the inner thread to generate the pressing load. The thread material's deformation is proportional to the load within the elastic range, but the load varies with the length of thread engagement; the torque makes the threads engaged mutually and thus limits its deformation, so within the length range of the thread engagement, the tensile load and the pressing load are a pair of opposite forces at each place.

The tightening torque leads to generate a pretightening force at the shoulder of the drill rod thread, and when the threads are pre-tightened to the yield point, the relation of the tightening torque versus the pre-tightening force is:

$$
T=F\left(p /(2 \pi)+\mu_{1} R_{t} / \cos (\beta / 2)+\mu_{1} d_{a} / 2\right)
$$

Where, $\mathrm{F}$ is the pre-tightening force, the initial load on the thread shoulder; $\mu 1$ is the friction coefficient between the contact faces of inner and outer threads, generally 0.13 $\sim 0.19 ; \mu 2$ is the friction coefficient between the contact faces of shoulders, generally $0.09 \sim 0.13$; Rt is the pitch diameter of the thread $\mathrm{i}$ within the length of engagement; $\mathrm{da}$ is the equivalent friction diameter of the shoulder toroid

The drill rod in operation will bear the tensile force F2 generated by the working torque $\mathrm{T}$ and the drill rod's dead weight. Under the joint action of the composite load, the residual pre-tightening force $\mathrm{F} 1$ occurs at the contact of the joints and is transmitted to the connecting threads via a number of threads so that the outer-thread joint suffers an axial tensile force and the inner-thread joint suffers an axial compressive force[6].

The external-load moment $\mathrm{T}$ equals the sum of the friction moment $\mathrm{T} 1$ between screw pair and the friction moment $\mathrm{T} 2$ between thread contact faces. According to the distribution of results from study of the axial loads on taper threads in Literature [7] and the friction moment 
between threads' contact faces, the formula as below can be obtained:

$$
T=T_{1}+T_{2}=\left[\left(F_{1}+F_{2}\right) \mathrm{dd}_{2}-2 \mathrm{ptan} \frac{\theta}{2} \mathrm{n}\right]\left(\tan \varphi+\tan \varphi_{\mathrm{v}}\right) / 2+f_{c} F_{1}\left(D_{0}^{3}-d_{0}^{3}\right) /\left[3\left(D_{0}^{2}-d_{0}^{2}\right)\right]
$$

Where, $\mathrm{P}$ is the thread intercept, $\mathrm{mm} ; \varphi$ is the helix angle, $\varphi=\tan \left(\mathrm{p} / \pi d_{2}\right) ; \varphi \mathrm{v}$ is the equivalent friction angle of the screw pair, $\varphi_{\mathrm{v}}=\operatorname{arc}\left(f_{s} / \cos \beta\right) ; \mathrm{d}_{2}^{\prime}$ is the pitch diameter of the thread at the start of the joint, $\mathrm{mm} ; \theta$ is the thread taper angle, $\left({ }^{\circ}\right) ; \beta$ is the tooth angle at the working face, 1:30 assumed; $f_{c}$ is the friction coefficient of the thread end face;D0 is the outer diameter of the annular end face at the thread root, $\mathrm{mm} ; \mathrm{d} 0$ is the inner diameter of the annular end face at the thread root, $\mathrm{mm}$.

In Literature [8], through finite element analysis of the drill rod joints, the stress at the connecting threads of the drill rod joints is determined, which is the largest at the first connecting thread and then reduce gradually at the threads gradually farther away from the shoulder.

\section{Build the Finite-Element-Analysis (FEA) Model of Drill Rods with Corrosion Defects}

During coal mine drilling, the drill rod mainly suffers the torque, axial force and bending moment, etc., in calculation through FEA, consideration needs to be given to geometric nonlinearity and material nonlinearity drill rods enjoy, and the followings are assumed:

- Ignore action of surrounding soil and rock masses against drill rods

- Materials enjoy plastic stress-strain characteristics

During the operation of the drill rod, thanks to the structural features of its joint and the long-term contact of the inner wall with corrosive medium, the end of its outer thread joint is vulnerable to corrosion. There are a variety of corrosion defects, for instance, spherical, cylindrical and rectangular defects, etc., in this paper, a 3D drill-rod joint model is used to constitute the spherical defect to study the impact of the change in defect depth and radius on stress at the corrosion defect, where the corroded depth in the drill rod is determined by referring to the corroded depth in pipes, not exceeding $60 \%$ of the wall thickness.

In this paper, the $\phi 73 \mathrm{~mm}$ drill rod specified in MT521-2006 Standard Drill Rods for Drilling in Coal Mine Gallery with the material parameters as shown in table 1 and the meshing of drill rod joints as shown in figure 1 .

Table 1. Parameters of materials of drill rods

\begin{tabular}{ccccc}
\hline $\begin{array}{c}\text { Drill } \\
\text { rod } \\
\text { size } \\
(\mathrm{mm})\end{array}$ & $\begin{array}{c}\text { Yield } \\
\text { strength } \\
(\mathrm{MPa})\end{array}$ & $\begin{array}{c}\text { Elastic } \\
\text { modulus } \\
(\mathrm{GPa})\end{array}$ & $\begin{array}{c}\text { Poisson's } \\
\text { ratio }\end{array}$ & $\begin{array}{c}\text { Friction } \\
\text { factor }\end{array}$ \\
\hline$\phi 73$ & 758 & 206 & 0.3 & 0.02 \\
\hline
\end{tabular}

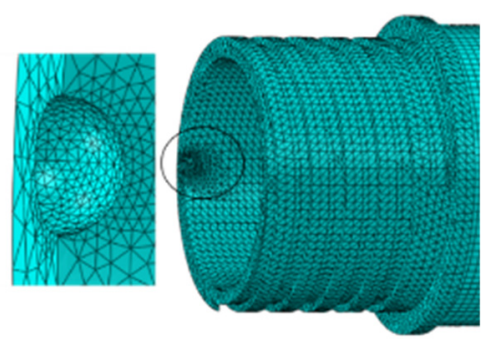

Figure 1. Meshing of corrosion defects in male joints.

To determine the value of the bending moment to be loaded, the regional expression of the ultimate bending moment capacity of pipes:

$$
M_{L}=4 R_{m}^{2} \mathrm{~s} \sigma_{u}
$$

Where, $\mathrm{s}$ is the thickness, $\mathrm{mm} ; \sigma_{\mathrm{u}}$ is the ultimate tensile strength, MPa; $\mathrm{R}_{\mathrm{m}}$ is the mean radius of the drill rod, $\mathrm{mm}$; $\mathrm{M}_{\mathrm{L}}$ is the ultimate bending moment capacity of an intact drill $\mathrm{rod}, \mathrm{N} \cdot \mathrm{mm}$.

With the above-mentioned formula, the bending moment borne by the drill rod can be approximately calculated, and the bending moment applied does no top $0.6 \mathrm{M}_{\mathrm{L}}$.

\section{FEA of Results}

\subsection{Analyze the stress at the corrosion defect of the drill rod under the bending moment}

By using FEA software, the torque and the axial force are exerted on the drill rod; considering the feeding force has a larger influence on the stress at the corrosion defects, the feeding force is exerted on one single end of it, and finally the bending moment is exerted on it. It is assumed that the bending moment is positive when the corrosion defect is under the compressive force, on the contrary, it is negative when the corrosion defect is under the tensile force, and the stress at the corrosion defect of the drill rod joint varies in size. When the corrosion defect is $2 \mathrm{~mm}$ in depth, $2 \mathrm{~mm}$ in radius and under the compressive force, the stress there is as shown in figure 2 and when it is under the tensile force, the stress there is as shown in figure 3 .

It is understood from figure 2 that, after exertion of the make-up torque, the working torque and the feeding force on the drill rod, the bending moment continues being exerted, the drill rod is first in the elastic stage during which the stress at the corrosion defect rises linearly, when the stress rises to $480 \mathrm{MPa}$, the bending moment rises within a certain range and the stress goes flattened, meaning the drill rod is in the yield stage; after the stress rises to $560 \mathrm{MPa}$, with the increasing bending moment loaded, the stress there slumped to $300 \mathrm{MPa}$, and then gets flat, and finally, with the continually increasing bending moment loaded, the stress there rockets up to $580 \mathrm{MPa}$. 


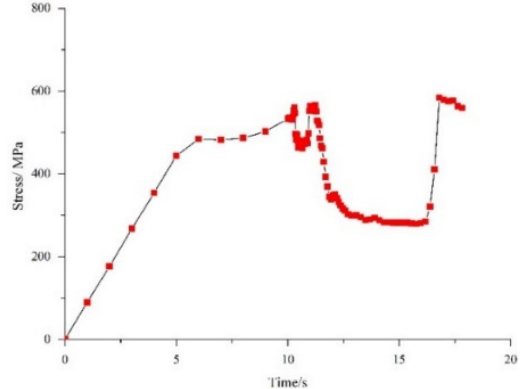

Figure 2. Change of stress at defects under positive bending moment

When the drill rod is under a positive bending moment, i.e. the corrosion defect under the compressive force, some stress there is counteracted, and with the loaded bending moment increasing with a certain range, its inhibition to the stress continues increasing.

It is understood from figure 3 that, the drill rod is first in the elastic stage during which the stress at the corrosion defect rises linearly, when the stress rises to $600 \mathrm{MPa}$, the bending moment rises within a certain range and the stress goes flattened, meaning the drill rod is in the yield stage; when the bending moment increases continually, the stress there continues rising linearly.

When the drill rod is under the negative bending moment, i.e. the stress there is under the tensile force, it drives the change of the stress there to rise, and it is known in combination with the relational expression of stress corrosion rate that the corrosion rate rises.

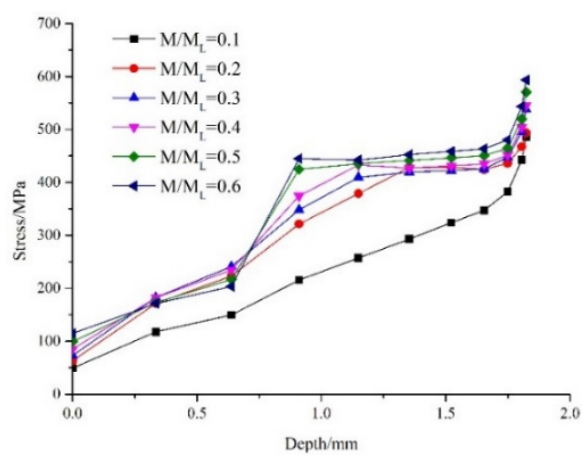

Figure 4. The relation of stress versus defect depth under the bending moment

It is observed from figure 4 that, when the same bending moment is exerted, the stress at the corrosion defect increases with its depth; in the case of the same depth of the corrosion defect and the exerted bending moment lower than $0.2 \mathrm{ML}$, the stress there increases with the bending moment, however, if the exerted bending moment is between $0.2 \mathrm{ML}$ and $0.6 \mathrm{ML}$, the stress there does not change clearly with the bending moment.

It is observed from figure 5 that, when the same bending moment is exerted, the stress at the corrosion defect increases with its radius; in the case of the same radius of the corrosion defect and the exerted bending moment lower than $0.3 \mathrm{ML}$, the stress there increases with the bending moment, however, if the exerted bending

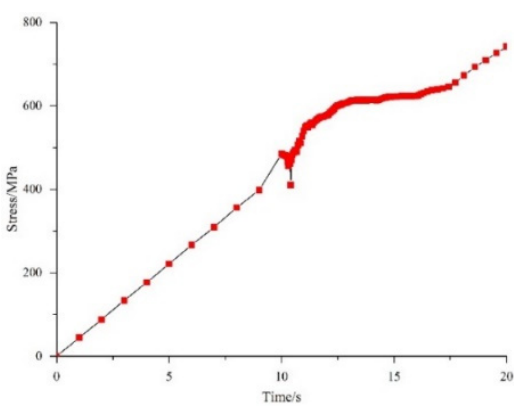

Figure 3. Change of stress at defects under negative bending moment

\subsection{Analyze the impact of corrosion-defect- related parameters}

To study the impact of the corrosion-defect-related parameters on the stress there, it is studied how the stress in the spherical corrosion defect changes along the route. The change relations of stress versus corrosion-defectrelated parameters along the route in the conditions that the drill rod is exerted with different bending moments are as shown in figure 4 and figure 5 .

It is observed from figure 4 that, when the same bending moment is exerted, the stress at the corrosion defect increases with its depth; in the case of the same depth of the corrosion defect and the exerted bending moment lower than $0.2 \mathrm{ML}$, the stress there increases with the bending moment, however, if the exerted bending moment is between $0.2 \mathrm{ML}$ and $0.6 \mathrm{ML}$, the stress there does not change clearly with the bending moment.

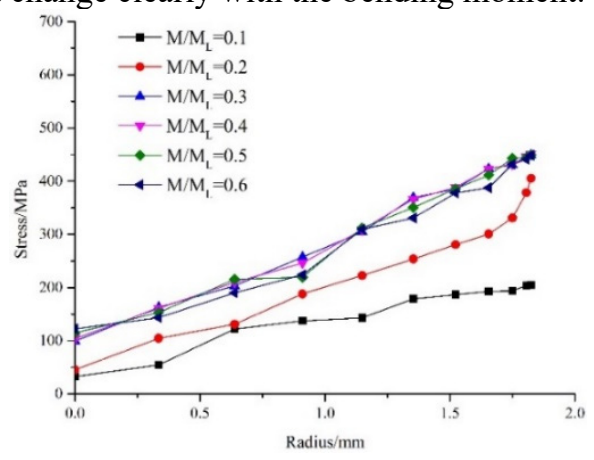

Figure 5. The relation of stress versus defect radius under the bending moment

moment is between $0.3 \mathrm{ML}$ and $0.6 \mathrm{ML}$, the stress there does not change clearly with the radius.

It is understood from comparison between figure 4 and figure 5 that, under the same bending moment, the slope of the curve of the stress versus depth of the corrosion defect is much higher than that of the curve of stress versus radius of the corrosion defect, therefore, the impact of the depth on the stress at the corrosion defect is much bigger than that of the radius on the stress. 


\section{Conclusion}

- In accordance with the theory on mechanochemical effect, the relation of stress versus corrosive rate under uniform corrosion is worked out when the drill rod is mainly under the torque, the feeding force and the bending moment, and building on this, that under local corrosion is worked out too;

- The results from loading of the bending moment indicate that, the compressive force, if exerted on the corrosion defect, plays a role to inhibit the stress at the corrosion defect to increase within a certain range; however, the tensile force, when exerted on the corrosion defect, will drive the stress there to increase, so making its corrosion rate faster;

- Study on corrosion-defect-related parameters shows, the change of the depth of the corrosion defect has an impact on stress there much larger than the radius; in addition, in the case that the corrosion-defect-related parameters are within a certain range, when the bending moment is loaded within a certain range, the stress at the corrosion defect does not change clearly.

\section{Acknowledgements}

This research was financially supported by the National Natural Science Foundation of China (Grant No. 51774320).

\section{References}

1. JIN Xiaohong, YANG Qi. Mechanical analysis or drill pipe under complex loads[J]. Mining \& Processing Equipment, 1997 (02): 16-18+49-50.

2. ZHU Li-juan, LIU Yong-gang, LI Fang-po, WANG Xin-hu, YUAN Jun-tao, LU Cai-hong. Corrosion Failure Reason of a G105 SteelDrillPipe[J]. Corrosion \& Protection, 2016, 37(9): 775.

3. TIAN Wei, YANG Zhuan-zhao, ZHAO Xue-hui, BAI Zhen-quan. Study on property of corrosion \& failure for S315 drill rod joints [J]. Physical Testing and Chemical Analysis part A: Physical Testing, 2008, 44(10): 575 578.

4. DONG Mengmeng. Development and application of $\Phi 73 \mathrm{~mm}$ high toughness and high strength drilling pipe[J]. Coal Geology \& Exploration, 2017, 45(2): 152-156.

5. TIAN Dong-Zhuang, SHI Zhi-Jun, GONG Cheng, DONG Meng-Meng. Research and Development on Drilling Pipe Matched for Horizontal Directional Drilling in Underground Mine[J]. Coal Science and Technology, 2013, 41(03): 24-27.

6. NIE Duzhong, MI Chengji. Influence of friction coefficients on connecting properties of taperthreaded joint of drill pipe[J]. Mining \& Processing Equipment, 2010, 38(11): 18-21.
7. Xi Juntong, Nie Gang, Mei Xuesong, Wu Xutang. Contact and connection behavior in finite element analysis of casing thread joints[J]. Journal of $\mathrm{Xi}^{\prime}$ an Jiaotong University, 1999(11):65-68.

8. ZHANG You zhen, SHI Zhi jun, TIAN Dong zhuang, GONG Cheng, PENG La mei. Finite element analysis and improved design for taper trapezium joint threads of high strength drilling rod with big hole[J]. Journal of China Coal Society, 2010,35(07):1219-1223. 